\section{Factores maternos relacionados con el peso al nacer de recién nacidos a término, Colombia, 2002-2011}

\author{
Maternal factors associated with birth weight in \\ term infants, Colombia, 2002-2011
}

\section{Fatores maternos associados ao peso ao nascer dos recém-nascidos a termo, Colômbia, 2002-2011}

\section{Resumen}

El estudio tuvo como objetivo identificar los factores maternos asociados con el peso al nacer, en Colombia, entre 2002-2011. Fue un estudio descriptivo, basado en información del Registro de Nacido Vivo de Colombia del Departamento Administrativo de Estadísticas Vitales, se clasificó el peso al nacer como: bajo peso al nacer < 2.500g, peso insuficiente 2.500 2.999g, peso adecuado 3.000-3.999g y macrosomía $\geq 4$.000g. Para el análisis se utilizó la U Mann-Whitney, Kruskall Wallis y un modelo de regresión logística multinomial. Las mujeres con mayor probabilidad de recién nacidos con bajo peso fueron las de 35 años o más (OR =1,4; IC95\%: 1,39$1,4)$, con bajo nivel educativo $(O R=1,1$; IC95\%: 1,1-1,1), solteras $(O R=$ 1,1; IC95\%: 1,1-1,2), sin asistencia a controles prenatales $(O R=1,9 ;$ IC95\%: 1,9-2,0) y de la zona rural (OR = 1,2; IC95\%: 1,1-1,2). Las mujeres con mayor prevalencia de recién nacidos macrosómicos fueron de 35 años o más (OR = 1,1; IC95\%: 1,1-1,1) y de 4 hijos o más (OR = 2,1; IC95\%: 2,0-2,1). El peso insuficiente tuvo un comportamiento similar al bajo peso al nacer. En conclusión, los factores sociodemográficos y maternos influencian el peso al nacer de recién nacidos de mujeres colombianas.

Peso al Nacer; Recién Nacido; Nutrición
Alejandro Estrada-Restrepo 1 Sandra Lucía Restrepo-Mesa 1 Natalia Del Carmen Ceballos Feria 1 Francisco Mardones Santander 2

doi: 10.1590/0102-311X00133215

$\begin{array}{ll}1 \text { Universidad de Antioquia, } & \text { Correspondencia } \\ \text { Antioquia, Colombia. } & \text { A. Estrada-Restrepo } \\ 2 \text { Universidad Católica de } & \text { Universidad de Antioquia. } \\ \text { Chile, Santiago, Chile. } & \text { Calle 67 \#53 108, Medellin, } \\ & \text { Antioquia 05001000, } \\ & \text { Colombia. } \\ & \text { alejandro.estrada@udea. } \\ & \text { edu.co }\end{array}$




\section{Introducción}

La Organización Mundial de la Salud (OMS) define el peso al nacer, como el primer peso del neonato, justo después del nacimiento, este es un importante indicador de salud pública que refleja las condiciones de salud fetal y neonatal, e indirectamente el estado nutricional materno; el peso al nacer determina la posibilidad del recién nacido de sobrevivir, y de tener un crecimiento sano 1,2 .

En el ámbito mundial, más de 20 millones de niños y niñas presentan bajo peso al nacer, no obstante, un $96 \%$ de estos nacimientos ocurren en países en vía de desarrollo; la prevalencia de bajo peso al nacer en Asia Meridional alcanza 31\%, en Asia Oriental y el Pacífico 7\%, en la India 40\%, África subsahariana $14 \%$, en Oriente Medio y África del Norte $15 \%$, estos resultados se han relacionado con altos niveles de pobreza 3 . En contraste, la mayor prevalencia de macrosomía se encuentra en países desarrollados y alcanza en las últimas dos décadas entre 15 y $25 \%$, debido principalmente al incremento de la obesidad materna y la diabetes gestacional 4 .

En América Latina la proporción de niños con bajo peso al nacer oscila entre 5,7\% y $12 \%$; Perú ocupa el primer lugar (11\%), seguido por Colombia, Paraguay y Venezuela (9\%). En el ámbito latinoamericano 85\% de las muertes neonatales están asociadas al bajo peso al nacer, nacimientos pretérmino y restricción del crecimiento fetal 5. En cuanto a la macrosomía, los países que presentan la mayor prevalencia son: Paraguay $(9,3 \%)$ y Cuba $(7,2 \%)$. Las menores prevalencias las presentan Ecuador (3,8\%) y Nicaragua (2,9\%) 4 .

El peso al nacer en Colombia tiene comportamientos diferenciales por departamento; las menores prevalencias de bajo peso al nacer se presentaron en Vaupés, Casanare, Guainía y Vichada, mientras Bogotá, Cundinamarca, Chocó, Antioquia, Boyacá y Atlántico presentaron las mayores prevalencias ${ }^{6}$. Los porcentajes más altos para este indicador se presentan en las ciudades con menores índices de pobreza y mayores índices de educación (Bogotá 12\%; Barranquilla y Cali 9\%) 7. Incluyendo nacimientos pretérmino y a término, el bajo peso al nacer en Colombia en el año 2000 fue 7,3\% y para el año 2011 alcanzó 9\% 6. En el país no se dispone de datos de macrosomía. En un estudio realizado en mujeres inmigrantes de diferentes países residentes en España se encontró que de un total de 8.544 mujeres colombianas, la macrosomía alcanzó 8,6\% 8 .

Otro indicador a considerar para analizar el peso al nacer, es el peso insuficiente, el cual incluye los recién nacidos entre 2.500 y $2.999 \mathrm{~g}$, este ha sido poco explorado como un indicador de salud pública y del cual no se cuenta con datos nacionales, ya que la atención se ha concentrado en el bajo peso 9,10. Sin embargo, existe evidencia científica de cómo, además del bajo peso, el peso insuficiente puede generar efectos deletéreos en etapas posteriores de la vida, como tener un menor potencial de crecimiento en la infancia ${ }^{11} \mathrm{y}$ mayor riesgo de sufrir patologías como: ictericia, infecciones, síndrome de dificultad respiratoria, problemas hematológicos, malformaciones congénitas y problemas metabólicos 12 .

Estudios realizados en diferentes hospitales de Brasil: Hospital Regional de Cotia (São Paulo) 9, Hospital Teresina (Piauí) 13, y en el estado de Pernambuco 14 han demostrado que el peso insuficiente ha alcanzado valores hasta del 25,7\%, 19,5\% y $31 \%$ respectivamente, lo que demuestra que en este país el peso insuficiente ha afectado $1 / 4$ de los recién nacidos en las últimas dos décadas. En el ámbito Nacional no se dispone de datos de peso insuficiente al nacer.

Existen múltiples factores que condicionan el peso al nacer, entre los que se encuentran el inadecuado acceso a los servicios de salud, el estado nutricional y de salud -antes y durante la gestación-, aspectos psicológicos, las condiciones económicas y sociales, bajo peso pregestacional, inadecuada ganancia de peso, ya sea por déficit o por exceso, déficit de micronutrientes antes y durante la gestación, inadecuado acceso y/o disponibilidad de alimentos, edad gestacional menor a 37 semanas, presencia de enfermedades como, anemia, hipertensión arterial, infecciones urinarias, diabetes gestacional, que condicionan el crecimiento intrauterino 15,16 .

Otros factores a considerar son de orden económico y social, como región o área de residencia materna, el nivel educativo, el estado civil, la edad materna, régimen de seguridad al que esta pertenece e inseguridad alimentaria ${ }^{17}$. En el caso de las adolescentes, se presenta mayor riesgo de recién nacido con bajo peso y peso insuficiente por aspectos como conductas de riesgo, en las que se incluye el consumo de alcohol, sustancias psicoactivas y el cigarrillo, inadecuados hábitos alimentarios, rechazo al embarazo y falta de redes de apoyo, por el contrario las mayores de 35 años tienen el mayor riesgo de macrosomía 18,19.

De acuerdo con lo anterior, el objetivo de este estudio fue identificar, a partir del registro de nacido vivo, los factores maternos asociados con el comportamiento del peso al nacer en Colombia, entre el 2002 y 2011. 


\section{Materiales y métodos}

Se realizó un estudio descriptivo, basado en información procedente del Registro de Nacido Vivo de Colombia, procedente del Departamento Administrativo de Estadísticas Vitales -DANE- que contiene datos sobre todos los nacimientos del país. Este registro es un documento legal, diligenciado por el médico o la enfermera que atendió el parto. Tiene como propósito unificar a nivel nacional la información relacionada con el nacimiento, además es fundamental para la inscripción de este en las oficinas autorizadas para el registro civil (Archivo Nacional de Datos, Departamento Administrativo Nacional de Estadística. Certificado de nacido vivo. http://190.25.231.249/metadatos/ index.php/catalog/233/vargrp/VG1, accedido el 31/Mar/2014); dentro de las variables que incluye este registro se encuentran datos generales como: datos antropométricos del recién nacido, información general de la madre y del parto (Ministerio de la Protección Social. Certificado de nacido vivo. https://www.dane.gov.co/files/investigaciones/ poblacion/defunciones/CnacidoVivo.pdf, accedido el 27/Feb/2014).

Fueron incluidos para este estudio los registros de nacido vivo entre 2002 y 2011. Después de 2011 se incluyeron todos los recién nacidos vivos a término ( $\geq 37$ semanas de gestación) de madres con embarazo de único feto, de nacionalidad colombiana, que tuvieron un peso al nacer entre 650 y $6.500 \mathrm{~g}$. Este rango fue utilizado después de hacer análisis en la base de datos general, y comprobar que los datos por debajo de $650 \mathrm{~g}$ y superiores a 6.500 g correspondían al $0,01 \%$ del total de nacimientos, lo que mostró que valores inferiores a estos eran de baja frecuencia, adicionalmente, los recién nacidos a término con estos pesos son considerados fisiológicamente excepcionales 20 .

La variable efecto fue definida como peso al nacimiento, clasificada en las siguientes categorías: bajo peso al nacer $<2.500 \mathrm{~g}$, peso insuficiente 2.500-2.999g, peso adecuado 3.000-3.999g y macrosomía $\geq 4.000 \mathrm{~g} 21$. Las variables explicativas fueron, año y región de nacimiento, área de residencia materna, edad, nivel educativo, régimen de seguridad social y estado civil de la madre, número de hijos nacidos vivos, número de consultas prenatales y sexo del neonato.

\section{Análisis de datos}

Se realizó un análisis descriptivo de las variables de estudio. Se estimó el porcentaje de peso al nacer en las categorías definidas previamente. Se emplearon las pruebas de U Mann-Whitney y Kruskall Wallis para comparar el peso al nacer con las variables cualitativas, prueba de chi cuadrado para comparar la clasificación de peso al nacer con los factores sociodemográficos y maternos. Se aplicó un modelo de regresión logística multinomial multivariado que tuvo como variable dependiente la clasificación de peso al nacer, y como independientes las variables de análisis; esta técnica permitió obtener los odds ratio (OR) para, bajo peso al nacer, peso insuficiente y macrosomía con sus respectivos intervalos de confianza al 95\% (IC95\%). Además, se efectuó un árbol de clasificación para determinar el comportamiento de la clasificación del peso al nacer, a partir de los factores sociodemográficos y maternos. Esta última técnica permitió valorar la interacción entre las variables independientes (los factores maternos) y cómo estos aumentan o disminuyen los porcentajes de bajo peso al nacer, peso insuficiente y macrosomía. El software empleado fue el IBM SPSS versión 21 (IBM Corp., Armonk, Estados Unidos).

\section{Aspectos éticos de la investigación}

Esta investigación siguió los principios éticos internacionales, Declaración de Helsinki, las directrices nacionales, Resolución no 008430 de octubre de 1993. A juicio de los investigadores no existió conflicto de intereses entre la instancia que suministró los datos y los investigadores. Para preservar la confidencialidad de los sujetos, en el análisis de los datos, no se consideró la identificación de la madre, ni del recién nacido. El proyecto fue aprobado por el Comité de Ética de la Facultad de Odontología de la Universidad de Antioquia, Colombia.

\section{Resultados}

En Colombia, en el año 2002 a 2011, se registraron 5.946.781 nacimientos a término, los cuales se distribuyeron por región, así: Atlántica 23,2\%, Central 24,7\%, Bogotá DC 18\%; Oriental 16,9\%, Pacífica 14,6\% y Orinoquía y Amazonía 2,5\%. El mayor número de nacimientos del país se presentó en la cabecera municipal $79,7 \%$, seguido del área rural disperso $12,6 \% \mathrm{y}$, finalmente, por el centro poblado con $7,7 \%$ (Tabla 1 ).

De acuerdo a los rangos de edad, las madres entre 20 y 34 años tuvieron $67,2 \%$ de los nacimientos, las menores de 20 años 22,8\%, y las mayores de 34 años 9,9\%. El nivel de estudios predominante fue básica-secundaria con $69,5 \%$. Tenían más de un hijo 56,8\% de las gestantes. Respecto a los controles prenatales, llama la atención que 3,5\% de las gestantes no asistió a ningún control prenatal, asistió entre uno y tres controles el $12,7 \%$, entre cuatro y seis $39,9 \%$, entre siete y ocho $30 \%$ y nueve y más controles $13,9 \%$ (Tabla 1). 
Tabla 1

Características maternas y sexo del recién nacido. Colombia, 2002-2011.

\begin{tabular}{|c|c|c|}
\hline \multirow[t]{2}{*}{ Características } & \multicolumn{2}{|c|}{ Total } \\
\hline & $\mathrm{n}$ & $\%$ \\
\hline \multicolumn{3}{|l|}{ Región } \\
\hline Atlántica & 1.381 .030 & 23,2 \\
\hline Oriental & 1.002 .469 & 16,9 \\
\hline Bogotá DC & 1.073 .179 & 18,0 \\
\hline Central & 1.468 .823 & 24,7 \\
\hline Pacífica & 869.659 & 14,6 \\
\hline Orinoquía y Amazonía & 151.621 & 2,5 \\
\hline Total & 5.946 .781 & 100,0 \\
\hline \multicolumn{3}{|c|}{ Área de residencia habitual de la madre } \\
\hline Cabecera municipal & 4.671 .025 & 79,7 \\
\hline Centro poblado & 452.836 & 7,7 \\
\hline Rural disperso & 736.087 & 12,6 \\
\hline Total & 5.859 .948 & 100,0 \\
\hline \multicolumn{3}{|l|}{ Edad de la madre (años) } \\
\hline$\leq 19$ años & 1.352 .402 & 22,8 \\
\hline $20-34$ & 3.981 .472 & 67,2 \\
\hline 35 y más & 591.631 & 9,9 \\
\hline Total & 5.925 .505 & 100,0 \\
\hline \multicolumn{3}{|l|}{ Estado conyugal de la madre } \\
\hline Soltera & 379.624 & 6,5 \\
\hline Casada/Unión libre & 3.303 .243 & 56,8 \\
\hline Separada/Divorciada/Viuda & 2.130 .619 & 36,6 \\
\hline Total & 5.813 .486 & 100,0 \\
\hline \multicolumn{3}{|c|}{ Número de consultas prenatales de la madre } \\
\hline Sin controles & 196.668 & 3,5 \\
\hline $1-3$ & 716.621 & 12,7 \\
\hline $4-6$ & 2.244 .280 & 39,9 \\
\hline $7-8$ & 1.688 .327 & 30,0 \\
\hline 9 y más & 780.381 & 13,9 \\
\hline Total & 5.626 .277 & 100,0 \\
\hline \multicolumn{3}{|c|}{ Número de hijos nacidos vivos a la largo de su vida } \\
\hline 1 & 2.548 .280 & 43,2 \\
\hline 2 & 1.761 .311 & 29,9 \\
\hline 3 & 878.446 & 14,9 \\
\hline 4 y más & 706.039 & 12,0 \\
\hline Total & 5.894 .076 & 100,0 \\
\hline \multicolumn{3}{|l|}{ Nivel de estudio de la madre } \\
\hline Ninguno/Preescolar/Primaria & 1.043 .929 & 17,8 \\
\hline Básica secundaria & 4.086 .858 & 69,5 \\
\hline Técnica/Tecnológica & 401.921 & 6,8 \\
\hline Profesional & 330.459 & 5,6 \\
\hline Posgrado & 16.502 & 0,3 \\
\hline Total & 5.879 .669 & 100,0 \\
\hline
\end{tabular}

(continua) 


\begin{tabular}{lcc} 
Características & $\mathbf{n}$ & Total \\
& & $\%$ \\
\hline Régimen de seguridad social en salud de la madre & 2.197 .371 & 37,8 \\
$\quad$ Contributivo & 2.389 .504 & 41,2 \\
Subsidiado & 869.422 & 15,0 \\
Vinculado/Excepción & 45.830 & 0,8 \\
Particular/Especial & 304.028 & 5,2 \\
No asegurado & 5.806 .155 & 100,0 \\
Total & & 51,3 \\
Sexo del recién nacido & 3.051 .949 & 48,7 \\
Hombre & 2.894 .832 & 100,0 \\
Mujer & 5.946 .781 & \\
Total & & \\
\hline
\end{tabular}

En este periodo de tiempo, el promedio de peso al nacer de los recién nacidos a término en el país fue $3.206 \pm 434$ g. Por otro lado, el porcentaje de bajo peso al nacer a término alcanzó $3,8 \%$, peso insuficiente $24,7 \%$ y la macrosomía $4,5 \%$. Al analizar el peso al nacer por año de nacimiento, se encontró que el bajo peso al nacer presentó variaciones entre 3,6\% y 4\%, mientras la macrosomía ha tenido un comportamiento con tendencia a la disminución entre $5,1 \%$ y $3,8 \%$. El peso insuficiente mostró una alta prevalencia oscilando entre $23,4 \%$ y $26,1 \%$, con tendencia al aumento en los últimos siete años del periodo de estudio. Llama la atención como el peso insuficiente supera el bajo peso al nacer en una relación de seis a uno y como la macrosomía presenta proporciones superiores al bajo peso al nacer, excepto en el año 2010 (Tabla 2).

La región que mayor proporción de bajo peso al nacer y peso insuficiente presentó fue Bogotá DC con $6,1 \%$ y $35,1 \%$, respectivamente. La Orinoquía y Amazonía presentó el mayor porcentaje de macrosomía (7,3\%). Por área de residencia, el peso adecuado fue similar y osciló entre $66,8 \%$ y $68,5 \%$, mientras que la macrosomía fue mayor en el centro poblado $(5,7 \%)$ (Tabla 2$)$.

La mayor proporción de bajo peso al nacer $(4,5 \%)$ y peso insuficiente $(28,3 \%)$, se presentó en las mujeres menores de 19 años, seguido el bajo peso de los recién nacidos de mujeres de 35 años y más $(4,1 \%)$, además los recién nacidos de estas mujeres fueron los que presentaron mayores porcentajes de macrosomía $(6,1 \%)$. De acuerdo al nivel educativo de la madre, los recién nacidos que presentaron mayores cifras de bajo peso al nacer $(4,2 \%)$ y peso insuficiente $(28,6 \%)$ fueron aquellas con nivel de estudio de posgrado, éste mismo grupo presentó la menor proporción de macrosomía; mientras aquellas sin ningún nivel educativo/preescolar/primaria presentaron la mayor proporción de recién nacidos con macrosomía $(5,2 \%)$ (Tabla 2$)$.

Las mujeres solteras tuvieron $4,8 \%$ de recién nacidos con bajo peso al nacer y $29,3 \%$ con peso insuficiente, por el contrario las mujeres casadas/unión libre y las separadas tuvieron mayor proporción de recién nacidos con macrosomía, $4,3 \%$ y $5,1 \%$, respectivamente (Tabla 2 ).

En las madres primigestantes se encontró porcentajes más elevados de bajo peso al nacer $(4,6 \%)$ y peso insuficiente $(28,1 \%)$, mientras que la macrosomía fue mayor en mujeres con 4 hijos o más (8\%). La mayor proporción de recién nacidos con bajo peso 5,2\% se encontró en aquellas madres que no asistieron a controles prenatales, las demás clasificaciones de peso al nacer tuvieron un comportamiento similar (Tabla 2).

Al realizar el análisis de regresión logística, todas las regiones tuvieron menor OR para bajo peso al nacer que Bogotá DC, donde la menor fue para los recién nacidos de la Orinoquía y Amazonía (OR = 0,3; IC95\%: 0,2-0,3), seguido de la región Oriental $(\mathrm{OR}=0,4$; IC95\% 0,3-0,4). Por otro lado, el mayor OR de recién nacidos macrosómicos fue para quienes nacieron en la región de la Orinoquía y Amazonía (OR = 4,3; IC95\%: 4,2-4,4), seguida de la región Atlántica (OR = 3,4; IC95\%: 3,3-3,5). Por área de residencia de la madre, aquellas del área rural disperso mostraron mayores OR de bajo peso al nacer (OR = 1,2; IC95\%: 1,1-1,2) (Tabla 3).

Las mujeres con mayores OR de recién nacidos con bajo peso al nacer fueron las pertenecientes al régimen subsidiado (OR = 1,1; IC95\%: 1,1-1,1), las solteras (OR = 1,1; IC95\%: 1,1-1,2), y aquellas 
Tabla 2

Características maternas y sexo del recién nacido, de acuerdo a la clasificación del peso al nacer. Colombia, $2002-2011$.

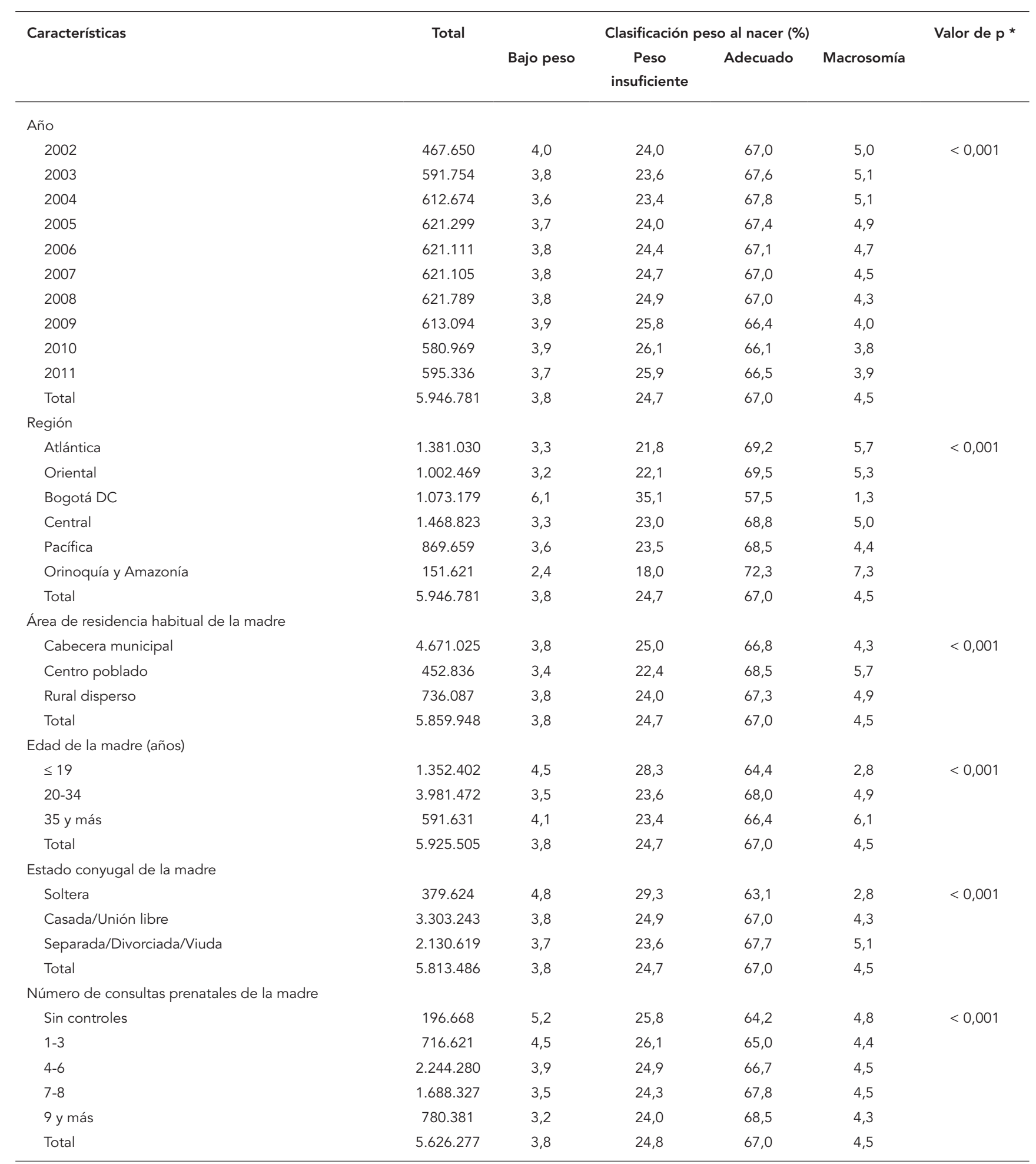

(continua) 
Tabla 2 (continuación)

\begin{tabular}{|c|c|c|c|c|c|c|}
\hline \multirow[t]{2}{*}{ Características } & \multirow[t]{2}{*}{ Total } & \multicolumn{4}{|c|}{ Clasificación peso al nacer (\%) } & \multirow[t]{2}{*}{ Valor de $\mathrm{p}$ * } \\
\hline & & Bajo peso & $\begin{array}{c}\text { Peso } \\
\text { insuficiente }\end{array}$ & Adecuado & Macrosomía & \\
\hline \multicolumn{7}{|c|}{ Número de hijos nacidos vivos a la largo de su vida } \\
\hline 1 & 2.548 .280 & 4,6 & 28,1 & 64,3 & 3,0 & $<0,001$ \\
\hline 2 & 1.761 .311 & 3,3 & 23,4 & 68,7 & 4,6 & \\
\hline 3 & 878.446 & 3,1 & 21,6 & 69,3 & 6,0 & \\
\hline 4 y más & 706.039 & 3,1 & 19,4 & 69,5 & 8,0 & \\
\hline Total & 5.894 .076 & 3,8 & 24,7 & 67,0 & 4,5 & \\
\hline \multicolumn{7}{|l|}{ Nivel de estudio de la madre } \\
\hline Ninguno/Preescolar/Primaria & 1.043 .929 & 3,8 & 23,7 & 67,3 & 5,2 & $<0,001$ \\
\hline Básica secundaria & 4.086 .858 & 3,8 & 24,8 & 66,9 & 4,4 & \\
\hline Técnica/Tecnológica & 401.921 & 3,8 & 25,4 & 66,7 & 4,1 & \\
\hline Profesional & 330.459 & 3,7 & 24,9 & 67,3 & 4,1 & \\
\hline Posgrado & 16.502 & 4,2 & 28,6 & 65,0 & 2,2 & \\
\hline Total & 5.879 .669 & 3,8 & 24,7 & 67,0 & 4,5 & \\
\hline \multicolumn{7}{|c|}{ Régimen de seguridad social en salud de la madre } \\
\hline Contributivo & 2.197 .371 & 3,8 & 25,5 & 66,7 & 4,0 & $<0,001$ \\
\hline Subsidiado & 2.389 .504 & 3,7 & 24,0 & 67,4 & 4,9 & \\
\hline Vinculado/Excepción & 869.422 & 3,8 & 24,0 & 67,3 & 4,9 & \\
\hline Particular/Especial & 45.830 & 3,9 & 23,5 & 67,4 & 5,2 & \\
\hline No asegurado & 304.028 & 4,3 & 26,5 & 65,1 & 4,0 & \\
\hline Total & 5.806 .155 & 3,8 & 24,7 & 67,0 & 4,5 & \\
\hline \multicolumn{7}{|l|}{ Sexo del niño } \\
\hline Hombre & 3.051 .949 & 3,1 & 21,2 & 69,9 & 5,7 & $<0,001$ \\
\hline Mujer & 2.894 .832 & 4,5 & 28,3 & 63,9 & 3,2 & \\
\hline Total & 5.946 .781 & 3,8 & 24,7 & 67,0 & 4,5 & \\
\hline
\end{tabular}

* Prueba de chi cuadrado.

Tabla 3

Asociación entre factores maternos y peso al nacer. Colombia, 2002-2011.

\begin{tabular}{|c|c|c|c|c|c|c|}
\hline \multirow[t]{2}{*}{ Características } & \multicolumn{2}{|c|}{ Bajo peso * } & \multicolumn{2}{|c|}{ Peso insuficiente * } & \multicolumn{2}{|c|}{ Macrosomía * } \\
\hline & OR (IC95\%) & Valor de $p$ & OR (IC95\%) & Valor de $p$ & OR (IC95\%) & Valor de $p$ \\
\hline \multicolumn{7}{|l|}{ Región } \\
\hline Bogotá DC & 1,00 & & 1,00 & & 1,00 & \\
\hline Atlántica & $0,40(0,40-0,41)$ & 0,00 & $0,50(0,50-0,50)$ & 0,00 & $3,40(3,38-3,52)$ & 0,00 \\
\hline Oriental & $0,40(0,38-0,39)$ & 0,00 & $0,50(0,49-0,50)$ & 0,00 & $3,30(3,21-3,34)$ & 0,00 \\
\hline Central & $0,40(0,40-0,41)$ & 0,00 & $0,50(0,51-0,52)$ & 0,00 & $3,10(3,08-3,21)$ & 0,00 \\
\hline Pacífica & $0,40(0,44-0,46)$ & 0,00 & $0,50(0,53-0,53)$ & 0,00 & $2,80(2,75-2,87)$ & 0,00 \\
\hline Orinoquía y Amazonía & $0,30(0,25-0,26)$ & 0,00 & $0,40(0,36-0,38)$ & 0,00 & $4,40(4,25-4,49)$ & 0,00 \\
\hline \multicolumn{7}{|c|}{ Área de residencia de la madre } \\
\hline Cabecera municipal & 1,00 & & 1,00 & & 1,00 & \\
\hline Centro poblado & $1,00(1,02-1,06)$ & 0,00 & $1,00(1,00-1,02)$ & 0,01 & $1,10(1,04-1,07)$ & 0,00 \\
\hline Rural disperso & $1,20(1,15-1,19)$ & 0,00 & $1,10(1,10-1,11)$ & 0,00 & $0,90(0,90-0,93)$ & 0,00 \\
\hline \multicolumn{7}{|l|}{ Edad de la madre (años) } \\
\hline $20-34$ & 1,00 & & 1,00 & & 1,00 & \\
\hline$\leq 19$ & $1,00(1,03-1,05)$ & 0,00 & $1,10(1,09-1,10)$ & 0,00 & $0,80(0,74-0,76)$ & 0,00 \\
\hline 35 y más & $1,40(1,39-1,43)$ & 0,00 & $1,10(1,10-1,12)$ & 0,00 & $1,10(1,08-1,11)$ & 0,00 \\
\hline
\end{tabular}

(continua) 
Tabla 3 (continuación)

\begin{tabular}{|c|c|c|c|c|c|c|}
\hline \multirow[t]{2}{*}{ Características } & \multicolumn{2}{|c|}{ Bajo peso * } & \multicolumn{2}{|c|}{ Peso insuficiente * } & \multicolumn{2}{|c|}{ Macrosomía * } \\
\hline & OR (IC95\%) & Valor de $\mathrm{p}$ & OR (IC95\%) & Valor de $p$ & OR (IC95\%) & Valor de $p$ \\
\hline \multicolumn{7}{|l|}{ Estado conyugal de la madre } \\
\hline Casada/Unión libre & 1,00 & & 1,00 & & 1,00 & \\
\hline Soltera & $1,10(1,11-1,15)$ & 0,00 & $1,10(1,07-1,09)$ & 0,00 & $0,90(0,87-0,91)$ & 0,00 \\
\hline Separada/Divorciada/Viuda & $1,00(0,96-0,99)$ & 0,00 & $1,00(0,97-0,98)$ & 0,00 & $1,00(1,03-1,05)$ & 0,00 \\
\hline \multicolumn{7}{|c|}{ Número de consultas prenatales de la madre } \\
\hline $7-8$ & 1,00 & & 1,00 & & 1,00 & \\
\hline Sin controles & $1,90(1,88-1,97)$ & 0,00 & $1,30(1,30-1,34)$ & 0,00 & $0,90(0,84-0,88)$ & 0,00 \\
\hline $1-3$ & $1,50(1,46-1,51)$ & 0,00 & $1,20(1,20-1,22)$ & 0,00 & $0,90(0,86-0,89)$ & 0,00 \\
\hline $4-6$ & $1,20(1,17-1,20)$ & 0,00 & $1,10(1,07-1,08)$ & 0,00 & $0,90(0,94-0,96)$ & 0,00 \\
\hline 9 y más & $0,80(0,82-0,85)$ & 0,00 & $0,90(0,91-0,92)$ & 0,00 & $1,00(1,02-1,05)$ & 0,00 \\
\hline \multicolumn{7}{|c|}{ Número de hijos nacidos vivos a la largo de } \\
\hline \multicolumn{7}{|l|}{ su vida } \\
\hline 1 & 1,00 & & 1,00 & & 1,00 & \\
\hline 4 y más & $0,50(0,50-0,52)$ & 0,00 & $0,60(0,63-0,64)$ & 0,00 & $2,10(2,03-2,09)$ & 0,00 \\
\hline 3 & $0,60(0,57-0,59)$ & 0,00 & $0,70(0,71-0,72)$ & 0,00 & $1,60(1,61-1,65)$ & 0,00 \\
\hline 2 & $0,60(0,63-0,65)$ & 0,00 & $0,80(0,78-0,79)$ & 0,00 & $1,30(1,33-1,36)$ & 0,00 \\
\hline \multicolumn{7}{|l|}{ Nivel de estudio de la madre } \\
\hline Básica secundaria & 1,00 & & 1,00 & & 1,00 & \\
\hline Ninguno/Preescolar/Primaria & $1,10(1,08-1,11)$ & 0,00 & $1,00(1,04-1,05)$ & 0,00 & $1,00(0,98-1,00)$ & 0,62 \\
\hline Técnica/Tecnológica & $0,90(0,93-0,97)$ & 0,00 & $1,00(0,97-0,99)$ & 0,00 & $1,00(0,97-1,00)$ & 0,91 \\
\hline Profesional & $0,90(0,87-0,91)$ & 0,00 & $0,90(0,94-0,96)$ & 0,00 & $1,00(0,96-1,00)$ & 0,60 \\
\hline Posgrado & $0,90(0,81-0,96)$ & 0,01 & $0,90(0,90-0,97)$ & 0,04 & $0,80(0,68-0,85)$ & 0,00 \\
\hline \multicolumn{7}{|l|}{ Régimen de seguridad social } \\
\hline Contributivo & 1,00 & & 1,00 & & 1,00 & \\
\hline No asegurado & $1,00(1,02-1,07)$ & 0,00 & $1,00(1,00-1,02)$ & 0,03 & $1,00(1,02-1,07)$ & 0,00 \\
\hline Subsidiado & $1,10(1,07-1,09)$ & 0,00 & $1,00(1,02-1,03)$ & 0,00 & $1,00(1,02-1,04)$ & 0,00 \\
\hline Vinculado/Excepción & $1,00(1,01-1,05)$ & 0,00 & $1,00(1,00-1,01)$ & 0,41 & $1,00(1,00-1,03)$ & 0,05 \\
\hline Particular/Especial & $1,10(1,03-1,14)$ & 0,01 & $1,00(0,97-1,02)$ & 0,89 & $1,10(1,01-1,11)$ & 0,02 \\
\hline \multicolumn{7}{|l|}{ Sexo } \\
\hline Mujer & 1,00 & & 1,00 & & 1,00 & \\
\hline Hombre & $0,60(0,62-0,63)$ & 0,00 & $0,70(0,67-0,68)$ & 0,00 & $1,70(1,64-1,67)$ & 0,00 \\
\hline \multicolumn{7}{|l|}{ Año } \\
\hline 2011 & 1,00 & & 1,00 & & 1,00 & \\
\hline 2002 & $1,00(0,99-1,04)$ & 0,13 & $0,90(0,90-0,92)$ & 0,00 & $1,30(1,22-1,28)$ & 0,00 \\
\hline 2003 & $1,00(0,97-1,02)$ & 0,62 & $0,90(0,90-0,92)$ & 0,00 & $1,20(1,20-1,26)$ & 0,00 \\
\hline 2004 & $1,00(0,95-0,99)$ & 0,02 & $0,90(0,90-0,92)$ & 0,00 & $1,20(1,22-1,27)$ & 0,00 \\
\hline 2005 & $1,00(0,97-1,02)$ & 0,59 & $0,90(0,93-0,95)$ & 0,00 & $1,20(1,16-1,21)$ & 0,00 \\
\hline 2006 & $1,00(1,02-1,07)$ & 0,00 & $1,00(0,95-0,97)$ & 0,00 & $1,10(1,10-1,15)$ & 0,00 \\
\hline 2007 & $1,00(1,02-1,06)$ & 0,00 & $1,00(0,96-0,98)$ & 0,00 & $1,10(1,09-1,14)$ & 0,00 \\
\hline 2008 & $1,00(0,98-1,02)$ & 0,73 & $1,00(0,94-0,96)$ & 0,00 & $1,10(1,08-1,12)$ & 0,00 \\
\hline 2009 & $1,00(1,02-1,06)$ & 0,00 & $1,00(0,99-1,01)$ & 0,89 & $1,00(1,00-1,04)$ & 0,03 \\
\hline 2010 & $1,10(1,04-1,08)$ & 0,00 & $1,00(1,00-1,02)$ & 0,02 & $1,00(0,97-1,01)$ & 0,24 \\
\hline
\end{tabular}

IC95\%: intervalo de confianza al 95\%; OR: odds ratio.

* La categoría de referencia del peso al nacer fue peso adecuado.

con nivel educativo a lo sumo primaria $(\mathrm{OR}=1,1$; IC95\%: 1,1-1,1). Las madres que no asistieron a controles prenatales presentaron la mayor razón de disparidades de bajo peso al nacer $(\mathrm{OR}=1,9$;
IC95\%: 1,9-2,0), evidenciándose un gradiente que muestra una disminución en el OR de bajo peso al nacer a mayor número de controles (Tabla 3 ). 
Las madres de 35 años y más presentaron las mayores razones de disparidad de recién nacidos con bajo peso al nacer (OR = 1,4; IC95\%: 1,39-1,43) y de macrosomía (OR = 1,1; IC95\%: 1,1-1,1). El tener más de un hijo reduce la razón de disparidades de bajo peso al nacer, por el contrario aumenta la de macrosomía, siendo las mujeres de 4 hijos y más las que tienen mayor riesgo $(\mathrm{OR}=2,1$; $\mathrm{IC} 95 \%$ : $2,0-2,1)$

Los recién nacidos de sexo masculino redujeron la razón de disparidades de bajo peso al nacer en $38 \%$ (OR = 0,6; IC95\%: 0,6-0,6), y presentaron los mayores OR de macrosomía (OR = 1,7; IC95\%: 1,6-1,7). El peso insuficiente presentó un comportamiento similar al del bajo peso al nacer. Por otro lado, las madres solteras redujeron la razón de disparidad de macrosomía en $15 \%(\mathrm{OR}=0,9$; IC95\%: 0,8-0,9) y las de nivel educativo de posgrado en $29 \%$ (OR = 0,7; IC95\%: 0,6-0,8). Por año de nacimiento, se observaron comportamientos similares en los OR para bajo peso al nacer y peso insuficiente, con respecto a la macrosomía, los OR fueron reduciéndose de forma leve, a medida que aumentó el tiempo (Tabla 3).

Después de aplicar un árbol de regresión, se encontró que el bajo peso al nacer se duplica $(16,6 \%)$, cuando la madre reside en Bogotá, con recién nacido de sexo femenino y realizó seis o menos controles prenatales; y aumentó en seis puntos porcentuales cuando residía en Bogotá, recién nacido de sexo masculino y asistió a seis o menos controles $(14,5 \%)$ (Figura 1a). Sin embargo, este porcentaje descendió cuando la madre vivía en otra región diferente a Bogotá, hijo de sexo masculino, dos o más hijos nacidos vivos a la largo de su vida y cuatro o más controles prenatales $(5,8 \%)$, así como en aquellas que sólo tenían un hijo vivo a lo largo de su vida y siete o más controles $(5,2 \%)$. Con relación al peso insuficiente, este aumentó alrededor de 13 puntos porcentuales en madres que residían en Bogotá y con hijo de sexo femenino (37,9\%). Esta proporción descendió aproximadamente siete puntos porcentuales, en madres de otras regiones diferentes a Bogotá, con hijo de sexo masculino y dos o más hijos nacidos vivos a lo largo de su vida (Figuras 1b y 1c).

En lo que respecta a la macrosomía, los mayores aumentos de este porcentaje se dieron cuando la madre residía en otras regiones diferentes a Bogotá, con hijo de sexo masculino, dos o más hijos a lo largo de su vida y tres o menos controles prenatales $(8,6 \%)$ o con cuatro o más controles $(7,8 \%)$. Por otro lado, los mayores descensos se encontraron en madres que residían en Bogotá, y con hijo de sexo femenino $(0,8 \%)$, (Figura 1).

\section{Discusión}

Los hallazgos de este estudio indicaron que factores maternos como encontrarse en los extremos de la edad reproductiva, el bajo nivel educativo de la madre, ser soltera, no asistir a controles prenatales y ser de la zona rural incrementan la probabilidad de tener recién nacido con bajo peso y peso insuficiente al nacer. La macrosomía se incrementó en la medida que aumenta el número de hijos y en aquellas mujeres con 35 años y más.

El presente estudio, al igual que otros de tipo retrospectivos realizados en bases de datos de países como: Taiwán 22 y Brasil 23 concluyen que la edad materna se asocia con el peso al nacer del neonato. El bajo peso al nacer se presenta en los extremos de la edad materna, mujeres adolescentes y mujeres añosas, mientras que la macrosomía es más prevalente en los extremos superiores de la edad 22,24. En este sentido, la Encuesta Nacional de Demografía y Salud (ENDS) de Colombia reportó en 2010 resultados similares a los del presente estudio, donde las mayores prevalencias de bajo peso al nacer en el país fueron en mujeres menores de 20 años y mayores de 357 .

Este estudio encontró asociación entre el nivel educativo y el peso al nacer, resultados similares se han reportado en diferentes latitudes como: EEUU 25, India 26 y España 27, al dar cuenta que, la educación materna contribuye favorablemente con los indicadores de salud, no sólo de la madre, sino del recién nacido en el periodo concepcional y post-natal. Un meta-análisis realizado por Silvestrin et al. 28 reportó que en Irán la diferencia en el bajo peso al nacer entre los hijos de mujeres sin educación, versus los recién nacidos de mujeres con mayor nivel educativo alcanzó 16,9\% y 5,4\%, respectivamente, el mismo estudio refiere que en Asia los niños de mujeres sin educación formal, versus las mujeres con secundaria o con un nivel educativo superior, presentan proporciones de $32 \%$ y $1,8 \%$ respectivamente de bajo peso al nacer.

Los recién nacidos de mujeres solteras presentaron mayor proporción de bajo peso al nacer. Resultados similares a los reportados por otros estudios a partir de bases de datos de registros de nacido vivo 29,30; el bajo peso del neonato en las mujeres solteras puede estar asociado con mayores limitaciones económicas y de acompañamiento en la gestación. Un estudio realizado en Colombia evidenció que $38,3 \%$ de las mujeres estan sin la presencia del compañero, situación que se agrava si las gestantes son adolescentes 18 .

No acudir a los controles prenatales, o asistir sólo entre uno y tres controles, se asoció con el bajo peso al nacer, resultados similares fueron encontrados en Brasil 31, China 32 e India 26, lo que 
Figura 1

Árbol de clasificación para peso al nacer en Colombia.

1a) Peso al nacer en recién nacidos.

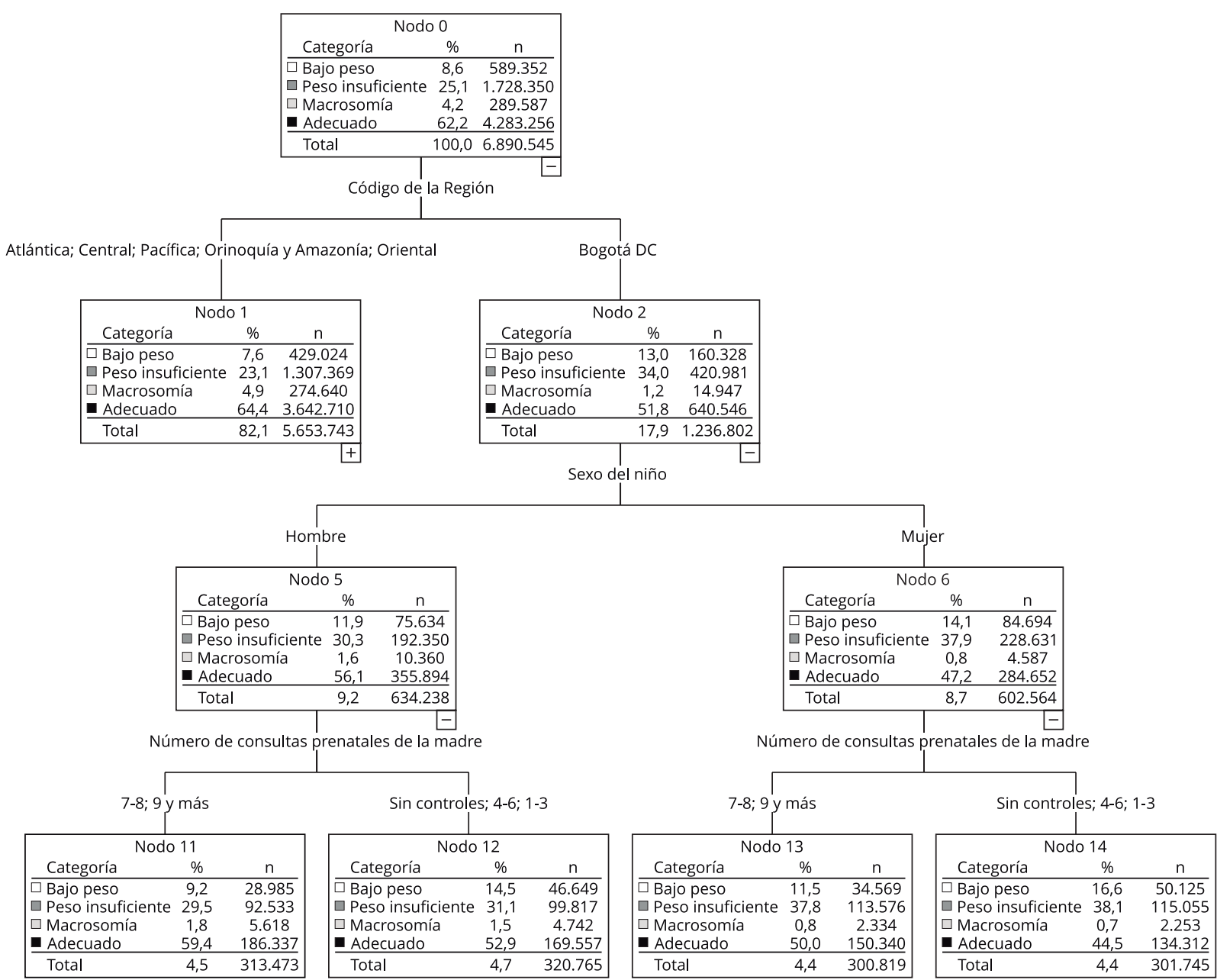

(continua)

demuestra la importancia del control prenatal para la detección oportuna de riesgos en la gestación. Aunado a lo anterior, en este estudio se encontró que vivir en la zona rural y pertenecer al régimen subsidiado incrementa el riesgo de bajo peso al nacer, lo que puede deberse a una mayor limitación en el acceso a los servicios de salud y a los programas de control prenatal e inseguridad alimentaria en el hogar, aspectos que condicionan el buen desarrollo de la gestación.

Los factores maternos asociados al peso insuficiente tuvieron un comportamiento similar al descrito para el bajo peso al nacer, no obstante, el peso insuficiente fue seis veces mayor que el bajo peso al nacer. En diferentes estudios se plantea que los neonatos con peso insuficiente, al igual que los menores de $2.500 \mathrm{~g}$, sufren adaptaciones ocasionadas por el déficit intrauterino, estos recién nacidos presentan alto riesgo de sufrir patologías como: ictericia, infecciones, síndrome de dificultad respiratoria, problemas hematológicos, malformaciones congénitas 9 , alteraciones fisiológicas y metabólicas que pueden ocasionar trastornos en etapas posteriores, que además de complicaciones posnatales, involucran alteraciones en el desarrollo 33 Adicionalmente, un estudio realizado por Minoru 
Figura 1 (continuación)

1b) Peso al nacer en recién nacidos de sexo masculino por regiones de Colombia.

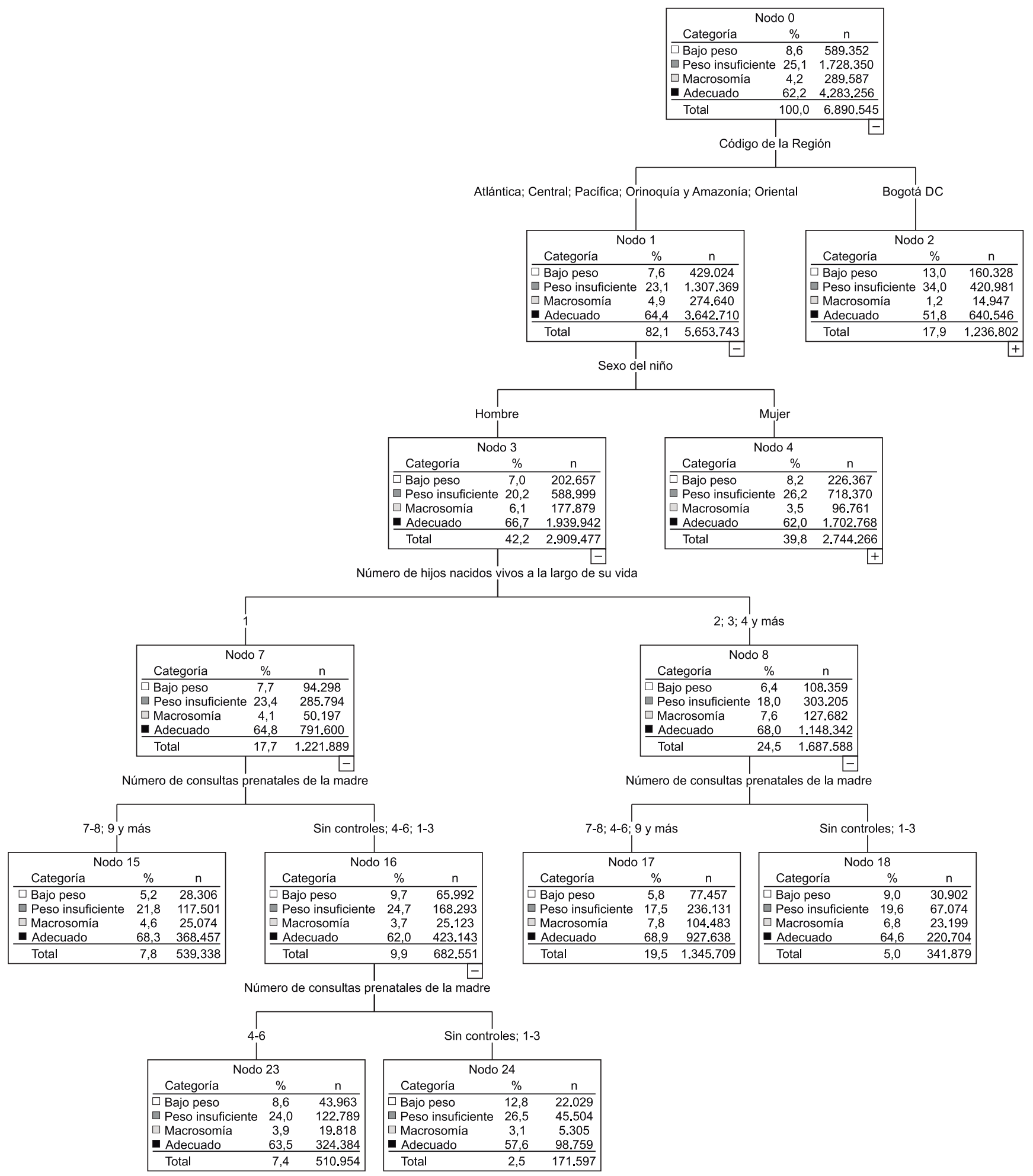


1c) Peso al nacer de los recién nacidos de sexo femenino por regiones de Colombia.

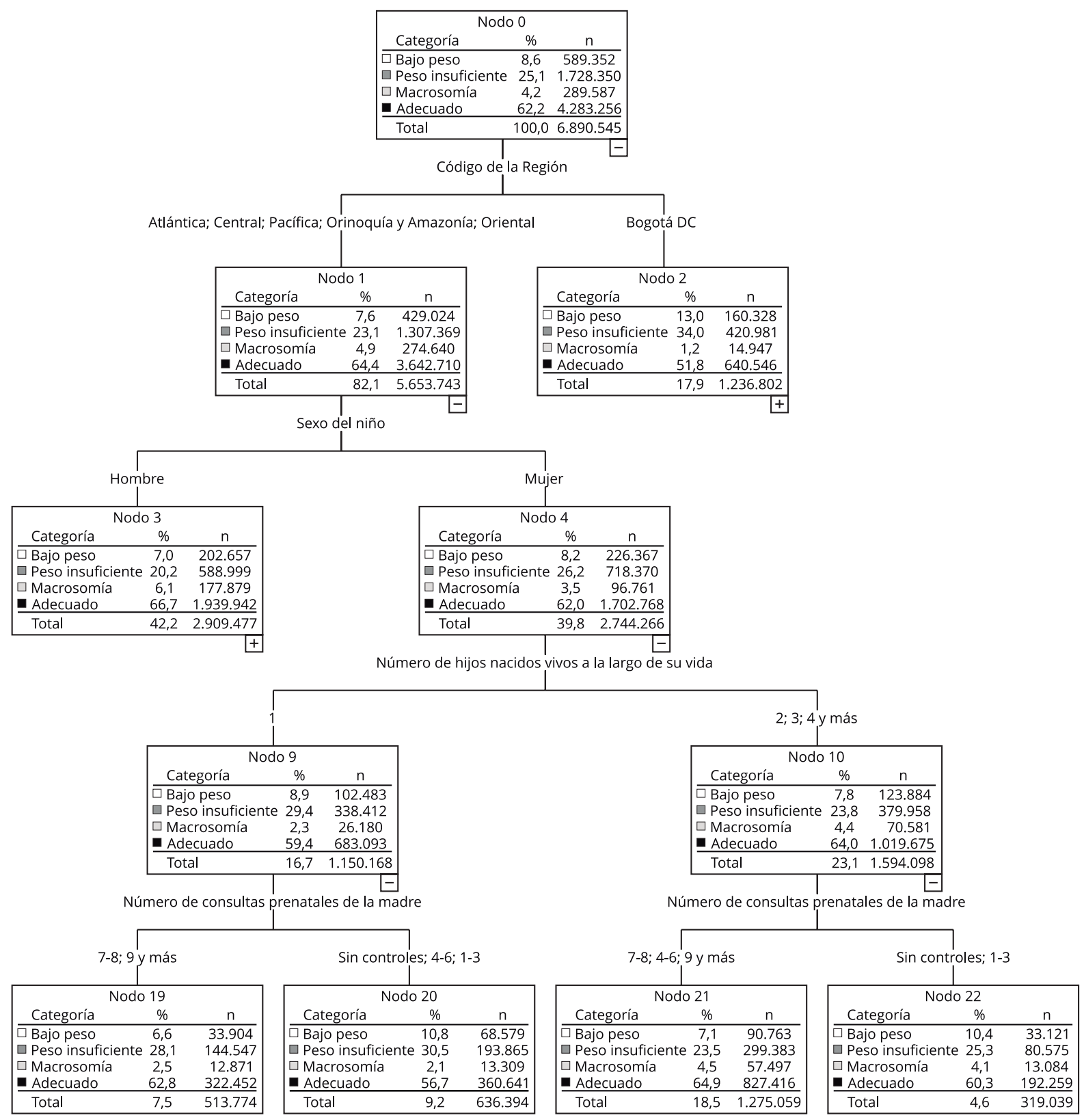

et al. 11 en Brasil concluye que los niños nacidos con peso insuficiente tienen una menor tasa de crecimiento, y un mayor riesgo de desarrollar un trastorno del crecimiento hasta la edad preescolar, sugiriendo un efecto negativo del peso insuficiente al nacer en el crecimiento infantil. Todos estos factores representan desventajas para la salud y productividad futura del individuo y el desarrollo económico y social de las comunidades

En relación a la paridad, se evidenció que en la medida que aumenta el número de hijos, se incrementa la proporción de macrosomía, resultados similares han reportado en Pakistán ${ }^{34}$, donde este fue el factor de riesgo principal para la macrosomía 
fetal. En muchos casos esta es consecuencia de la obesidad materna antes de la gestación y/o de la excesiva ganancia de peso durante el embarazo 35 . La Encuesta Nacional de la Situación Nutricional en Colombia (ENSIN 2010) reportó que, 24,8\% de las madres con sobrepeso y $9,8 \%$ con obesidad, es decir, 34,6\% presentó algún grado de exceso de peso para la edad gestacional, con mayor proporción de exceso de peso en las mujeres entre 37 y 49 años en las cuales alcanzó $44 \%$ 36, esto demuestra la gran proporción de mujeres que tienen algún grado de exceso de peso en el país durante la gestación y que predispone al recién nacido a padecer macrosomía al nacer.

La evidencia muestra que los hijos de madres con sobrepeso $\mathrm{u}$ obesidad tienen mayor cantidad de masa grasa al nacimiento 37 que puede generar defectos congénitos, complicaciones en el parto, tanto para el recién nacido, como para la madre 38 , y predispone a la obesidad y enfermedades crónicas en la infancia y en la adultez 39,40 .

$\mathrm{Al}$ realizar el análisis por regiones del país, se encontró que la mayor proporción de macrosomía reportada fue en la región de la Orinoquía y Amazonía, esto puede guardar relación con el exceso de peso en la población adulta que según la ENSIN 2010 alcanza el 54,2\% en los departamentos de esta región (Vichada, Caquetá, Guaviare y Guanía) 36.

El bajo peso al nacer tiene múltiples implicaciones no sólo biológicas, sino también económicas, sociales y políticas, se ha relacionado con la nutrición y su importancia en los primeros mil días de vida, periodo denominado la ventana de oportunidad para el desarrollo que inicia desde la preconcepción hasta los primeros dos años 41 . En la serie Lancet de 2008, los autores afirman la asociación que tiene una adecuada nutrición gestacional y en los dos primeros años, con el desarrollo del capital humano ${ }^{2}$. Los niños que crecen desnutridos son más propensos a tener una talla más baja, logros educativos más reducidos y menores ingresos económicos en la edad adulta; las mujeres, en especial, están condicionadas a tener hijos pequeños y con inadecuado peso al nacer 38 .

El indicador biológico más importante del crecimiento y desarrollo intrauterino es el peso al nacer, por lo anterior es imperante que en el país, además de la vigilancia del bajo peso al nacer, se consideren como indicadores de salud pública el peso insuficiente y la macrosomía al nacer, por las consecuencias que ambos tienen en el periodo prenatal y posnatal para la salud de la madre y del recién nacido. Los programas de control prenatal, además de las actividades de atención y tratamiento, deben desarrollar acciones de promoción de la salud que orienten y sensibilicen a las futuras madres sobre la importancia de los cuidados prenatales para favorecer el peso al nacer del neonato.

Debe enfatizarse la vigilancia nutricional y la detección oportuna de mujeres en riesgo, para que por medio de un adecuado acompañamiento de los profesionales de la salud alcancen ganancias de peso, ajustadas a su estado nutricional preconcepcional, y de acuerdo con ello, favorecer el peso del recién nacido. Los recién nacidos con bajo peso, peso insuficiente o macrosomía, requieren en muchos casos un manejo especializado que demanda altos costos en salud, que en países como Colombia podrían invertirse en el control de los riesgos asociados a estos indicadores y en la resolución de sus causas estructurales para contribuir a romper el círculo de la desnutrición materno-fetal.

Dentro de las limitaciones del presente estudio, se resaltan: el no contar con datos de peso materno preconcepcional y durante la gestación, estilos de vida de la madre, complicaciones del embarazo, entre ellas, el retraso de crecimiento intrauterino, estrato socioeconómico, así como la ausencia del número de semanas de gestación en algunos de los registros de nacimiento. Otra de las limitaciones fue la calidad de la información utilizada, en cuanto a la completitud y la validación de algunos de los datos. El haber trabajado con partos a término y de un solo feto, puede restringir algunas comparaciones con estadísticas nacionales previas. Además, a pesar que en poblaciones similares a la nuestra se evaluaban niños pretérmino y con restricción del crecimiento intrauterino, pero con factores maternos similares a los nuestros, el no haber controlado por algunas variables -entre ellas la edad gestacional- no permiten valorar el efecto modificador de esta sobre el peso al nacer, lo que puede limitar la comparación de algunos de nuestros resultados con otras publicaciones. 


\section{Colaboradores}

Todos los autores participaron en las diferentes etapas de escritura del artículo.

\section{Agradecimientos}

Esta publicación es resultado de la investigación realizada con el apoyo financiero de la Estrategia de Sostenibilidad 2014-2015, de la Universidad de Antioquia. Los investigadores agradecen además a dicho grupo, y al Centro de Investigaciones de la Facultad Nacional de Salud Pública, el haber posibilitado el acceso a las bases de datos de recién nacidos del DANE 2002-2011.

\section{Referencias}

1. World Health Organization. World health statistics 2005. http://www.who.int/gho/publications/ world_health_statistics/whostat2005en.pdf?ua=1 (accedido el 09/Mar/2014).

2. Victora C, Adair L, Fall C, Hallal P, Martorell R, Richter L, et al. Maternal and child undernutrition: consequences for adulthealth and human capital. Lancet 2008 ; 371:340-57.

3. Fondo para la Infancia de las Naciones Unidas. Progreso para la infancia: un balance sobre la nutrición. http://www.unicef.org/spanish/progress forchildren/2006n4/files/PFC4_SP_8X11.pdf (accedido el 31/Mar/2014).

4. Koyanagi A, Zhang J, Dagvadorj A, Hirayama F, Shibuya K, Souza JP, et al. Macrosomia in 23 developing countries: an analysis of a multicountry, facility-based, cross-sectional survey. Lancet 2013; 381:476-83.

5. Instituto Nacional de Salud. Informe del evento bajo peso al nacer a término, periodo epidemiológico 1 al 10 del año 2013. Comportamiento del evento en América. http://www.ins.gov.co/lineas-de-accion/ Subdireccion-Vigilancia/Informe\%20de\%20Even to\%20Epidemiologico/BAJO\%20PESO\%20AL\%20 NACER\%20\%202013.pdf (accedido el 21/Abr/2015).

6. Así Vamos en Salud. Análisis: prevalencia de bajo peso al nacer - georreferenciado. http://www.asi vamosensalud.org/inidicadores/estado-de-salud/ grafica.ver/14 (accedido el 04/Mar/2014).

7. Asociación Probienestar de la Familia Colombiana; Ministerio de la Protección Social; Instituto Colombiano de Bienestar Familiar; United States Agency for International Development. Encuesta Nacional de Demografía y Salud: ENDS-2010. Bogotá: Asociación Probienestar de la Familia Colombiana; 2011.

8. Restrepo S, Estrada A, Gonzalez L, Agudelo A. Newborn birth weights and related factors of native and immigrant residents of Spain. J Immigr Minor Health 2015; 17:339-48.
9. Costa R, Caldevilla D, Gallo P, Figueiredo B, Leone C. Incidência e características dos recém-nascidos de peso insuficiente de uma coorte de neonatos de um hospital público regional de área metropolitana. Rev Bras Crescimento Desenvolv Hum 2013; 23:238-43.

10. Rincón Benjumea MV, Gallestey Bacallao J, Jiménez R. La predicción del bajo peso y del peso insuficiente al nacer mediante antropometría materna. Revista Hacia la Promoción de la Salud 2009; 14:35-53.

11. Minoru R, Oliveira D, Abreu L, Leone C. Peso insuficiente ao nascer e crescimento alcançado na idade pré-escolar, por crianças atendidas em creches filantrópicas do município de Santo André, São Paulo, Brasil. Rev Bras Saúde Matern Infant 2009; 9:477-85.

12. Bhuiyan A, Chen W, Srinivasan S, Azevedo M, Berenson G. Relationship of low birth weight to pulsatile arterial function in asymptomatic younger adults: the Bogalusa Heart Study. Am J Hypertens 2010; 23:168-73.

13. Lima G, Sampaio H. Influencia dos fatores obstétricos, socioeconômicos e nutricionais da gestante sobre o peso do recém-nascido: estudo realizado em uma maternidade em Teresina, Piauí. Rev Bras Saúde Matern Infant 2004; 44:253-261.

14. Rocha J. Baixo peso, peso insuficiente e peso adequado ao nascer, em 5.940 nascidos vivos na cidade do Recife. J Pediatr (Rio J.) 1991; 67:297-304.

15. Zablah R. Bajo peso al nacer y desnutrición. Rev GASTROHNUP 2011; 13:157-9.

16. Fajardo Luig R, Cruz Hernández J, Gómez Sosa E, Isla Valdés A, Hernández García P. Factores de riesgo de bajo peso al nacer, estudio de tres años en el municipio Centro Habana. Rev Cuba Med Gen Integr 2008; 24:1-17.

17. Delgado H, Monteagudo S, Rodríguez D, Vega M, Sotolongo M. Estratificación del bajo peso al nacer desde un enfoque de determinantes sociales. Finlay $2013 ; 3: 42-53$. 
18. Zapata-Lopéz N, Restrepo-Mesa SL. Factores asociados con el índice de masa corporal materno en un grupo de gestantes adolescentes, Medellín, Colombia. Cad Saúde Pública 2013; 29:921-34.

19. Restrepo-Mesa SL, Zapata-López N, Parra Sosa BE, Escudero Vásquez LE, Atalah E. Embarazo adolescente: características maternas y su asociación con el peso al nacer del neonato. Arch Latinoam Nutr 2014; 64:99-107.

20. Instituto Nacional de Salud. Informe del evento mortalidad perinatal y neonatal tardía, hasta el periodo epidemiológico 12 del año 2012. Bogotá DC: Instituto Nacional de Salud; 2012.

21. Organización Mundial de la Salud. El estado físico: uso e interpretación de la antropometría. Ginebra: Organización Mundial de la Salud; 1995. (Serie de Informes Tecnicos, 854).

22. Weng Y, Yang C, Chiu Y. Risk assessment of adverse birth outcomes in relation to maternal age. PLoS One 2014; 9:e114843.

23. Veloso HJ, Silva AA, Bettiol H, Goldani MZ, Lamy Filho F, Simões VM, et al. Low birth weight in São Luís, northeastern Brazil: trends and associated factors. BMC Pregnancy Childbirth 2014; 14:155.

24. Toirac Lamarque AS, Pascual López V, Martínez Jiménez A, Area Suárez RI. Macrosomía fetal en madres no diabéticas. Caracterización mínima. Medisan 2013; 17:6052-62.

25. Gage T, Fang F, O'Neill E, DiRienzo G. Maternal education, birth weight, and infant mortality in the United States. Demography 2013; 50:615-35.

26. Kader M, Perera N. Socio-economic and nutritional determinants of low birth weight in India. North Am J Med Sci 2014; 6:302-8.

27. Juárez S, Revuelta B. Socioeconomic differences in low birth weight: revisiting epidemiological approaches. Revista Espanola de Investigaciones Sociológicas 2013; 144:73-95.

28. Silvestrin S, Silva C, Hirakata V, Goldani A, Silveira P, Goldani M. Maternal education level and low birth weight: a meta-analysis. J Pediatr (Rio J.) 2013; 89:339-45.

29. Shah P, Zao J, Ali S. Maternal marital status and birth outcomes: a systematic review and metaanalyses. Matern Child Health J 2011; 15:1097-109.

30. Marlon F, Márquez J, Vargas E, Quiroga G, Pinzón G. Análisis del bajo peso al nacer en Colombia 20052009. Rev Salud Pública 2013; 15:577-88.
31. Branco-da-Fonseca C, Louzada M, Carvalho L, Fiorini R. Adequacy of antenatal care and its relationship with low birth weight in Botucatu, São Paulo, Brazil: a case-control study. BMC Pregnancy Childbirth 2014; 14:255.

32. Dai L-L, Mao Y-Y, Luo X-M, Shen Y-P. Prenatal care in combination with maternal educational level has a synergetic effect on the risk of neonatal low birth weight: new findings in a retrospective cohort study in Kunshan city, China. PLoS One 2014; 9:e113377.

33. Restrepo-Mesa SL, Estrada-Restrepo A, GonzálezZapata LI, Agudelo-Suarez AA, Ronda-Pérez E. Peso al nacer: una comparación de sus factores relacionados entre los recién nacidos de madres españolas y madres colombianas residentes en España. Arch Latinoam Nutr 2010; 60:15-22.

34. Ali H, Ishtiaq S. Fetal macrosomia: its maternal and neonatal complications. Professional Medical Journal 2014; 21:421-6.

35. Mardones F, Bove I, Domínguez A. Asociaciones entre el crecimiento prenatal y la antropometría materna en el Uruguay. Nutr Hosp 2014; 30:643-9.

36. Profamilia; Instituto Nacional de Salud; Instituto Colombiano de Bienestar Familiar; Ministerio de Protección Social. Encuesta nacional de la situación nutricional en Colombia 2010: ENSIN. Bogotá: Instituto Colombiano de Bienestar Familiar; 2011.

37. Ladino L, Moreno R, Campoy C. Nutrición materna y sus implicaciones en la adiposidad infantil. Revista Salud Bosque 2014; 4:27-34.

38. Victora C. Los mil días de oportunidad para intervenciones nutricionales. De la concepción a los dos años de vida. Arch Argent Pediatr 2012; 110:311-7.

39. Loaiza S, Coustasse A, Urrutia-Rojas X, Atalah E. Birth weight and obesity risk at first grade in a cohort of Chilean children. Nutr Hosp 2011; 26:214-9.

40. Mardones F, Villaroel L, Karzulovic L, Barja S, Arnaiz $\mathrm{P}$, Taibo M, et al. Association of perinatal factors and obesity in 6- to 8-year-old Chilean children. Int J Epidemiol 2008; 37:902-10.

41. Save the Children. Nutrition in the first 1,000 days: State of the World's Mothers 2012. Fairfield: Save the Children; 2012. 


\section{Abstract}

The study aimed to identify maternal factors associated with birth weight in Colombia from 2002 to 2011. This was a descriptive study based on data from the Live Birth Registry of Colombia, Administrative Department of Vital Statistics. Birth weight was classified as low birth weight $<2,500 \mathrm{~g}$, insufficient birth weight 2,500-2,999g, normal birth weight 3,000-3,999g, and high birth weight $\geq 4,000$ g. Data analysis used MannWhitney U test, Kruskal-Wallis test, and multinomial logistic regression. Women with increased likelihood of low birth weight newborns were 35 years or older $(\mathrm{OR}=$ 1.4; 95\%CI: 1.39-1.4), had little schooling $(O R=1.1$; 95\%CI: 1.1-1.1), were single (OR = 1.1; 95\%CI: 1.1-1.2), without prenatal care $(O R=1.9 ; 95 \% C I: 1.9-2.0)$, and lived in rural areas $(O R=1.2$; 95\%CI: 1.1-1.2). Women with higher prevalence of high birth weight newborns were 35 years or older (OR =1.1; 95\%CI: 1.1-1.1) and had four or more children $(O R=2.1 ; 95 \% C I$ 2.0-2.1) Insufficient birth weight showed a similar pattern to low birth weight. In conclusion, social, demographic, and maternal factors influence the birth weight of newborns in Colombia.

Birth Weight; Newborn Infant; Nutrition

\section{Resumo}

Este estudo objetiva identificar os fatores maternos associados ao peso ao nascer, em Colômbia, no período de 2002 a 2011. Foi um estudo descritivo, baseado em informações do Registro de Nascidos Vivos de Colômbia do Departamento Administrativo de Estatísticas Vitais; se classificou o peso ao nascer como baixo peso ao nascer < 2.500g; peso insuficiente 2.500-2.999g, peso adequado 3.000-3.999g e macrossomia $\geq 4.000 \mathrm{~g}$. Para a análise foi usada a U Mann-Whitney, KruskalWallis e um modelo de regressão logística multinomial. As mulheres com maior probabilidade de recémnascidos com baixo peso foram as de 35 anos ou mais $(O R=1,4$; IC95\%: 1,39-1,4), com baixo nivel escolar $(O R=1,1$; IC95\%: 1,1-1,1), solteiras $(O R=1,1$; IC95\%: $1,1-1,2)$, sem assistência a controles pré-natais $(O R=$ 1,9; IC95\%: 1,9-2,0) e da zona rural (OR = 1,2; IC95\%: 1,1-1,2). As mulheres com maior prevalência de recém-nascidos macrossômicos foram de 35 anos ou mais $(O R=1,1$; IC95\%: 1,1-1,1) e com 4 filhos ou mais $(O R=2,1 ;$ IC95\%: 2,0-2,1). O peso insuficente teve um comportamento similar ao baixo peso ao nascer. Em conclusão, os fatores sociodemográficos e maternos in fluenciam o peso ao nascer do recém-nascido das mulheres colombianas.

Peso ao Nascer; Recém-Nascido; Nutrição
Recibido el 22/Ago/2015

Verisón final presentada el 10/Feb/2016 Aprobado el 12/Feb/2016 